\title{
Effect of Upstream Pressure on the Flux of Single Permeant in Pervaporation
}

\author{
J. BaI, A. E. Fouda, and Takesi MatsuUra ${ }^{*, \dagger}$ \\ Institute for Environmental Chemistry, National Research Council of Canada, \\ Ottawa, Canada, K1A 0R6 \\ *Industrial Membrane Research Institute, Department of Chemical Engineering, \\ University of Ottawa, Ottawa, Canada, K1N $6 \mathrm{N5}$
}

(Received November 15, 1993)

\begin{abstract}
The effect of the upstream pressure on the pervaporation flux of water was studied both theoretically and experimentally. The increase in the pervaporation flux with an increase in the upstream pressure was predicted using newly developed transport equations that allow the pressure change across the membrane and include both liquid and vapor phase transport. The conventional solution-diffusion model, on the other hand, fails to predict the effect of the upstream pressure, particularly when an assumption is made that the pressure of the permeant inside the membrane is constant across the membrane and is equal to the upstream pressure. The pervaporation flux of water through a polydimethysiloxane membrane and an aromatic polyamide membrane was found, experimentally, to increase with an increase in the upstream pressure.

KEY WORDS Pervaporation / Membrane / Aromatic Polyamide /

Polydimethylsiloxane / Membrane Transport /
\end{abstract}

Pervaporation has been recognized recently as one of the most versatile membrane separation processes. Fundamental research on pervaporation has been carried out intensively parallel to the growth of the number of industrial applications. At present the majority of studies on pervaporation transport is based on the solution-diffusion model ${ }^{1-5}$ with few exceptions that are based on the pore model. ${ }^{6-8}$ Although the transport equations derived from both models explain the effect of the downstream pressure on pervaporation flux equally well, they come to different conclusions regarding the effect of the upstream pressure; i.e., the solution-diffusion model predicts little effect of the upstream pressure, when the downstream pressure is nearly equal to zero, while the pore model predicts that the pervaporation flux should change linearly with an increase in the upstream pressure. There are also two different kinds of experimental data. Whereas Greenlaw et al. and Paul and Ebralima concluded that the pervaporation flux remained constant with an increase of the upstream pressure for the system hexane/ polyethylene and cyclohexane/highly swollen cross-linked rubbery membrane, ${ }^{5,9}$ respectively, Okada and Matsuura showed that the flux increased linearly for the system ethyl alcohol/heptane mixture in cellulose membranes of different pore sizes. ${ }^{6}$

It has to be noted that the solution-diffusion mechanism does not necessarily predict no upstream pressure effect. The above prediction can only be made under an assumption that the pressure of the permeant remains the same as the upstream pressure across the membrane. When the above assumption is removed, the change of the pervaporation flux is predicted with a change in the upstream pressure. It is

\footnotetext{
† To whom correspondence should be addressed.
} 
obvious, therefore, from above discussions that no conclusive evidence has been obtained so far either theoretically or experimentally about the effect of upstream pressure on pervaporation flux.

It is the objective of this work to study the effect of the upstream pressure on the pervaporation flux in detail, both theoretically and experimentally. In particular, pervaporation experiments are conducted for the permeation of water through polydimethylsiloxane and aromatic polyamide membranes. While the former membrane represents rubbery hydrophobic membranes, the latter represents glassy hydrophilic membranes.

\section{EXPERIMENTAL}

\section{Membranes}

A casting solution was prepared by mixing $10.3 \mathrm{wt} \%$ aromatic polyamide (poly- $m$-phenylene-iso(70)-co-tere(30)-phthalamide, abbreviated as APA), $3.8 \mathrm{wt} \% \mathrm{LiCl}$, and 85.9 $w t \%$ dimethyl acetamide. The filtered and air bubble free casting solution was cast onto a glass plate by a casting knife to the thickness of $5.08 \times 10^{-5} \mathrm{~m}$ at room temperature and kept in an oven at $95^{\circ} \mathrm{C}$ for two hours. The membrane looked completely dry and transparent after a long evaporation period and is considered to be dense and symmetric. Then, the membrane was immersed in ice cold water to be removed from the glass plate. A commercial polydimethylsiloxane (PDMS) membrane of thickness $5.08 \times 10^{-5} \mathrm{~m}$ was supplied by General Electric Co.

\section{Pervaporation Experiments}

The pervaporation system consists of a static permeation cell made of stainless steel and with an effective membrane area of $9.6 \times 10^{-4} \mathrm{~m}^{2}$, permeate collection traps and a vacuum pump. About $100 \mathrm{~g}$ of feed liquid was loaded in the cell at room temperature $\left(23^{\circ} \mathrm{C}\right)$ and nitrogen pressure was applied to the feed liquid from a nitrogen cylinder. This pressure is called hereafter upstream pressure. Then vacuum was applied on the permeate side by turning on the vacuum pump. The pressure of the permeate side was adjusted to a predetermined value by introducing dry air stream into the vacuum line. This pressure is called hereafter downstream pressure. The downstream pressure can also be adjusted to a predetermined value by controlling a pressure control valve installed between the permeate collection trap and the vacuum pump. It was confirmed experimentally that the pervaporation flux at a given downstream pressure did not depend on whether it was achieved by introducing dry air stream or by adjusting the control valve. The ranges of the upstream and downstream pressures were $1.01 \times 10^{5}-1.14 \times 10^{6} \mathrm{~Pa}(0-$ $150 \mathrm{psig})$ and $400-4000 \mathrm{~Pa}(3-30 \mathrm{mmHg})$, respectively. The feed liquid was water in most cases except few experiments with an aqueous acetic acid solution of $0.08 \mathrm{~mol}$ fraction. The permeate product was condensed in the permeate collection trap cooled by liquid nitrogen. Stable permeation rates were reached usually within 120 minutes after permeate collection was started. The permeation rate was determined by weighing the cold trap filled with the permeate. The composition of the permeate sample was analyzed using gas chromatograph when the feed was an aqueous acetic acid solution.

\section{Reverse Osmosis}

Reverse osmosis experiments were conducted also by using a static permeation cell. About $150 \mathrm{~g}$ of feed aqueous sodium chloride solution $(1700 \mathrm{ppm})$ was loaded in the cell at room temperature and nitrogen pressure was applied to the feed liquid to adjust the feed pressure to $1825008 \mathrm{~Pa}(250 \mathrm{psig})$. The permeate side was maintained at an atmospheric pressure. The first five $\mathrm{ml}$ of the permeate was discarded before the permeate sample collection was started. The permeation rate was determined by weighing the permeate sample collected during a predetermined period. The 
sodium chloride concentration of the feed and the product sample was analyzed by conductivity measurement. The solute separation, $f$, was defined as,

$$
f=\frac{\text { feed }_{\mathrm{NaCl} \mathrm{ppm}}-\text { permeate }_{\mathrm{NaCl} \mathrm{ppm}}}{\text { feed }_{\mathrm{NaCl} \mathrm{ppm}}}
$$

\section{Scanning Electron Microscope}

Selected samples of the membranes were prepared for the scanning electron microscope (SEM) observations. Cross sections of the membranes were prepared by quenching in liquid nitrogen, then breaking without damaging the membrane. The sample was then attached to the carbon holder and shadowed with a thin gold layer to prevent any charging by the electron beam. The membrane section was subjected to a narrow beam of electrons with kinetic energies of $1-25 \mathrm{kV}$ causing the liberation of low energy electrons from the atoms in the surface which create the image on the micrograph.

\section{THEORETICAL}

Some transport equations are developed for a pervaporation system where the transport of a single component is involved.

The pervaporation flux through a membrane segment of thickness $\mathrm{d} x$ is related to the chemical potential gradient by

$$
J=-\frac{c_{\mathrm{m}}}{f_{\mathrm{m}}} \frac{\mathrm{d} \mu}{\mathrm{d} x}
$$

where $c_{\mathrm{m}}$ and $f_{\mathrm{m}}$ are the permeant concentration in the membrane $\left(\mathrm{mol} \mathrm{m}^{-3}\right)$ and the friction $\left(J \mathrm{~s} \mathrm{~m}^{-2} \mathrm{~mol}^{-1}\right)$ to the movement of the permeant in the membrane, respectively. ${ }^{10}$

\section{Liquid Phase Transport}

At the upstream face of the membrane the permeant in the membrane is in equilibrium with the feed liquid under the upstream pressure, $p_{2}$. Therefore, the chemical potential of

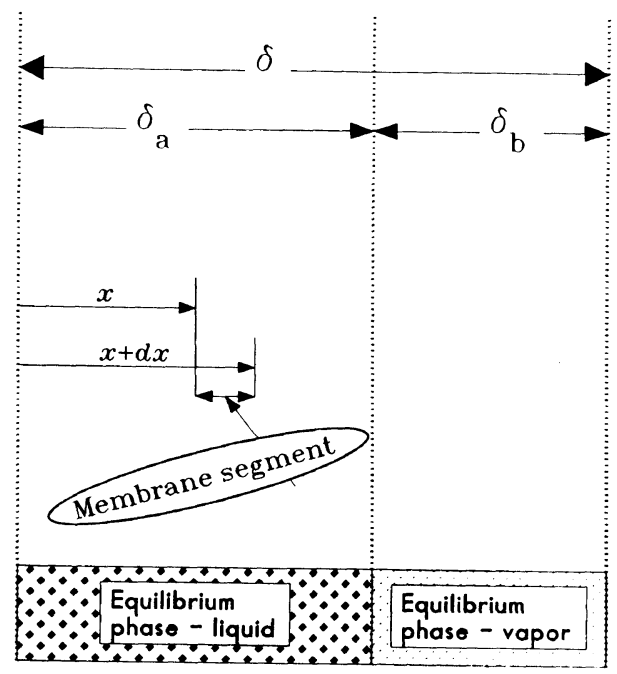

Figure 1. Schematic illustration of the imaginary liquid and vapor phase that are in thermodynamic equilibrium with the permeant in the membrane.

the permeant in the membrane is equal to that of the feed liquid which can be written as,

$$
\mu=\mu^{*}+v\left(p_{2}-p_{*}\right)
$$

where $\mu^{*}$ is the chemical potential of liquid at the saturation vapor pressure, $p_{*}$, and $v$ is the molar volume of the feed liquid.

At a distance $x$ from the upstream interface, a membrane segment whose thickness is $\mathrm{d} x$ is considered (see Figure 1). (It would be possible to assume the presence of an imaginary pure liquid phase which is in equilibrium with the permeant in the membrane segment when the distance $x$ is not large.) An attempt is made in the following to calculate the chemical potential change $d \mu$ in the above imaginary pure liquid phase.

The pressure of the pure liquid phase changes from $p$ to $p+\mathrm{d} p$ as the distance increases from $x$ to $x+\mathrm{d} x, \mathrm{~d} p$ having a negative sign. Since the molar volume of the permeant is constant, the change in the chemical potential of the permeant from distance $x$ to $x+\mathrm{d} x$ should be,

$$
\mathrm{d} \mu=v \mathrm{~d} p
$$


Although the above equation was developed for the imaginary pure liquid phase, it should also be applicable to the membrane phase. The pressure of the imaginary pure liquid phase continues to decrease as the distance $x$ increases, and at a distance $\delta_{\mathrm{a}}$, the pressure becomes the saturation vapor pressure. Since the concentration of the permeant in the membrane, $c_{\mathrm{m}}$, can be assumed to be constant from $x=0$ to $x=\delta_{\mathrm{a}}$ (Lonsdale made the same assumption when he developed his reverse osmosis transport equations ${ }^{11}$ ), eq 2 can be integrated into,

$$
J \int_{0}^{\delta_{\mathrm{a}}} \mathrm{d} x=\frac{-c_{\mathrm{m}}}{f_{\mathrm{m}}} v \int_{p_{2}}^{p_{*}} \mathrm{~d} p
$$

Therefore,

$$
J=\frac{c_{\mathrm{m}}}{f_{\mathrm{m}}} v \frac{\left(p_{2}-p_{*}\right)}{\delta_{\mathrm{a}}}
$$

An assumption was made in the above integration that $f_{\mathrm{m}}$ is also unchanged throughout the integration. As for $c_{\mathrm{m}}$, the following relationship was established by Greenlaw et al. between $c_{\mathrm{m}}$ and the activity, ${ }^{5}$

$$
c_{\mathrm{m}}=\sigma a+\tau a^{\mathrm{m}}
$$

The activity used in the above equation is considered to be that of imaginary pure liquid phase, hence $a=1$. Then,

$$
c_{\mathrm{m}}=\sigma+\tau
$$

As for $f_{\mathrm{m}}$, it is related to the diffusion coefficient $D_{\mathrm{m}}$ by,

$$
f_{\mathrm{m}}=\frac{R T}{D_{\mathrm{m}}}
$$

Furthermore, Greenlaw et al. has given the relation between $D_{\mathrm{m}}$ and $c_{\mathrm{m}}$ as, ${ }^{5}$

$$
D_{\mathrm{m}}=D_{\mathrm{m} 0}\left(1+\alpha c_{\mathrm{m}}^{n}\right)
$$

Combining eq $6,8,9$, and 10 ,

$$
J=\frac{\left(D_{\mathrm{m} 0} / R T\right)(\sigma+\tau)\left(1+\alpha(\sigma+\tau)^{n}\right) v}{\delta_{\mathrm{a}}}\left(p_{2}-p_{*}\right)
$$

\section{Vapor Phase Transport}

From the distance $x=\delta_{\mathrm{a}}$ to $x=\delta$ the equilibrium phase is considered to be in the vapor phase (see Figure 1) and the pressure changes from $p_{*}$ to $p_{3}$. The change in the chemical potential in a small segment $\mathrm{d} x$ can be written as,

$$
\mathrm{d} \mu=\frac{R T}{p} \mathrm{~d} p
$$

assuming ideal gas low. Thus, the chemical potential gradient is established by eq 4 and 12, respectively, in the liquid transport and vapor transport region. Then, eq 2 is written as,

$$
\begin{aligned}
J & =-\frac{c_{\mathrm{m}}}{f_{\mathrm{m}}} \frac{\mathrm{d} \mu}{\mathrm{d} x} \\
& =-\frac{c_{\mathrm{m}}}{f_{\mathrm{m}}} \frac{R T}{p} \frac{\mathrm{d} p}{\mathrm{~d} x}
\end{aligned}
$$

Rearranging,

$$
J \mathrm{~d} x=-\frac{c_{\mathrm{m}} R T}{f_{\mathrm{m}}} \frac{\mathrm{d} p}{p}
$$

Since the activity of the vapor in the equilibrium phase can be written as,

$$
a=p / p_{*}
$$

Combination of eq $7,9,10,15$, and 16 yields,

$$
J \mathrm{~d} x=\frac{-\left(\sigma\left(p / p_{*}\right)+\tau\left(p / p_{*}\right)^{m}\right) R T}{\left[R T / D_{\mathrm{m} 0}\left(1+\alpha\left(\sigma\left(p / p_{*}\right)+\tau\left(p / p_{*}\right)^{m}\right)^{n}\right]\right.} \frac{\mathrm{d}\left(p / p_{*}\right)}{\left(p / p_{*}\right)}
$$

Rearranging, 


$$
J \mathrm{~d} x=-\frac{D_{\mathrm{m} 0}\left(\sigma\left(p / p_{*}\right)+\tau\left(p / p_{*}\right)^{m}\right)\left(1+\alpha\left(\sigma\left(p / p_{*}\right)+\tau\left(p / p_{*}\right)^{m}\right)^{n}\right)}{\left(p / p_{*}\right)} \mathrm{d}\left(p / p_{*}\right)
$$

Setting $\zeta=p / p_{*}$,

$$
J \mathrm{~d} x=-\frac{D_{\mathrm{m} 0}\left(\sigma \zeta+\tau \zeta^{m}\right)\left(1+\alpha\left(\sigma \zeta+\tau \zeta^{m}\right)^{n}\right)}{\zeta} \mathrm{d} \zeta
$$

Integrating from $x=\delta_{\mathrm{a}}$ to $x=\delta$,

$$
J \int_{\delta_{\mathbf{a}}}^{\delta} \mathrm{d} x=-\int_{p_{*} / p_{*}}^{\zeta_{3}} \frac{D_{\mathrm{m} 0}\left(\sigma \zeta+\tau \zeta^{m}\right)\left(1+\alpha\left(\sigma \zeta+\tau \zeta^{m}\right)^{n}\right)}{\zeta} \mathrm{d} \zeta
$$

and,

$$
J=\frac{\int_{\zeta_{3}}^{1} \frac{D_{\mathrm{m} 0}\left(\sigma \zeta+\tau \zeta^{m}\right)\left(1+\alpha\left(\sigma \zeta+\tau \zeta^{m}\right)^{n}\right)}{\zeta}}{\delta_{\mathrm{b}}} \mathrm{d} \zeta
$$

where $\zeta_{3}=p_{3} / p_{*}$ and $\delta_{\mathrm{b}}=\delta-\delta_{\mathrm{a}}$.

Combination of Liquid Phase and Vapor Phase Transport.

From eq 11,

$$
\delta_{\mathrm{a}}=\frac{\left(D_{\mathrm{m} 0} / R T\right)(\sigma+\tau)\left(1+\alpha(\sigma+\tau)^{n}\right) v}{J}\left(p_{2}-p_{*}\right)
$$

and from eq 21 ,

$$
\delta_{\mathrm{b}}=\frac{\int_{\zeta_{3}}^{1} \frac{D_{\mathrm{m} 0}\left(\sigma \zeta+\tau \zeta^{m}\right)\left(1+\alpha\left(\sigma \zeta+\tau \zeta^{m}\right)^{n}\right)}{\zeta} \mathrm{d} \zeta}{J}
$$

Since $\delta=\delta_{\mathrm{a}}+\delta_{\mathrm{b}}$

$$
\delta=\frac{\left(D_{\mathrm{m} 0} / R T\right)(\sigma+\tau)\left(1+\alpha(\sigma+\tau)^{n}\right) v}{J}\left(p_{2}-p_{*}\right)+\frac{\int_{\zeta_{3}}^{1} \frac{D_{\mathrm{m} 0}\left(\sigma \zeta+\tau \zeta^{m}\right)\left(1+\alpha\left(\sigma \zeta+\tau \zeta^{m}\right)^{n}\right)}{\zeta} \mathrm{d} \zeta}{J}
$$

Therefore,

$$
J=\frac{\left(D_{\mathrm{m} 0} / R T\right)(\sigma+\tau)\left(1+\alpha(\sigma+\tau)^{n}\right) v}{\delta}\left(p_{2}-p_{*}\right)+\frac{\int_{\zeta_{3}}^{1} \frac{D_{\mathrm{m} 0}\left(\sigma \zeta+\tau \zeta^{m}\right)\left(1+\alpha\left(\sigma \zeta+\tau \zeta^{m}\right)^{n}\right)}{\zeta} \mathrm{d} \zeta}{\delta}
$$

The above equation is valid when $p_{2} \leq p_{*}$. When $p_{3}>p_{*}$ the imaginary equilibrium phase is liquid across the membrane, and the flux should be written as,

$$
J=\frac{\left(D_{\mathrm{m} 0} / R T\right)(\sigma+\tau)\left(1+\alpha(\sigma+\tau)^{n}\right) v}{\delta}\left(p_{2}-p_{3}\right)
$$


Equations 25 and 26 give explicitly the effect of the upstream pressure and the downstream pressure on the pervaporation rate. (Note that this approach is closely related to Lonsdales' approach for reverse osmosis transport and allows the change in the permeant pressure across the membrane.) The main features of the transport equations are:

1. The transport mechanism includes the pressure change across the membrane.

2. The transport mechanism includes both liquid transport and vapor transport.

Greenlaw et al. ${ }^{5}$ and Lee $^{12}$ discussed the pervaporation transport using the solutiondiffusion model with an assumption that the pressure of the permeant inside the membrane is constant and equal to the upstream pressure across the membrane. In particular, Greenlaw et al. have shown explicitly the effect of the upstream and the downstream pressure on the pervaporation flux and calculated the flux numerically under different conditions using the parameters listed in Table I. The results are summarized in Figure 2. The same numerical parameters were used in eq 25 and 26 and results illustrated in Figure 3. While Greenlaw et al. predict no upstream pressure effect on pervaporation flux at $p_{3}=0$, the flux increases linearly with an increase in the upstream pressure, $p_{2}$, according to the newly developed transport equations. The linear relationship was also predicted by the transport equations based on the pore flow model. ${ }^{6}$

Table I. Greenlaw parameters for calculating the pervaporation flux at different upstream and downstream pressures

\begin{tabular}{llll}
\hline Parameter & & \multicolumn{1}{c}{ Value } & Units \\
\hline$D_{\mathrm{m} 0}$ & $=24.2 \times 10^{-13}$ & $\mathrm{~m}^{2} \mathrm{~s}^{-1}$ \\
$\alpha$ & $=0.001788$ & \\
$n$ & $=1.33$ & \\
$m$ & $=3.4$ & \\
$\tau$ & $=545.3$ & $\mathrm{~mol} \mathrm{~m}^{-3}$ \\
$\sigma$ & $=137.9$ & $\mathrm{~mol} \mathrm{~m}^{-3}$ \\
$p_{*}$ & $=24918$ & $\mathrm{~Pa}^{3}$ \\
$v$ & $=130.58 \times 10^{-6}$ & $\mathrm{~m}^{3} \mathrm{~mol}^{-1}$ \\
\hline
\end{tabular}

Kataoka et $a l .{ }^{13}$ discussed the effect of the upstream pressure on the pervaporation (at downstream pressure $=0 \mathrm{~Pa}$ ) and the reverse osmosis flux (at downstream pressure $=$

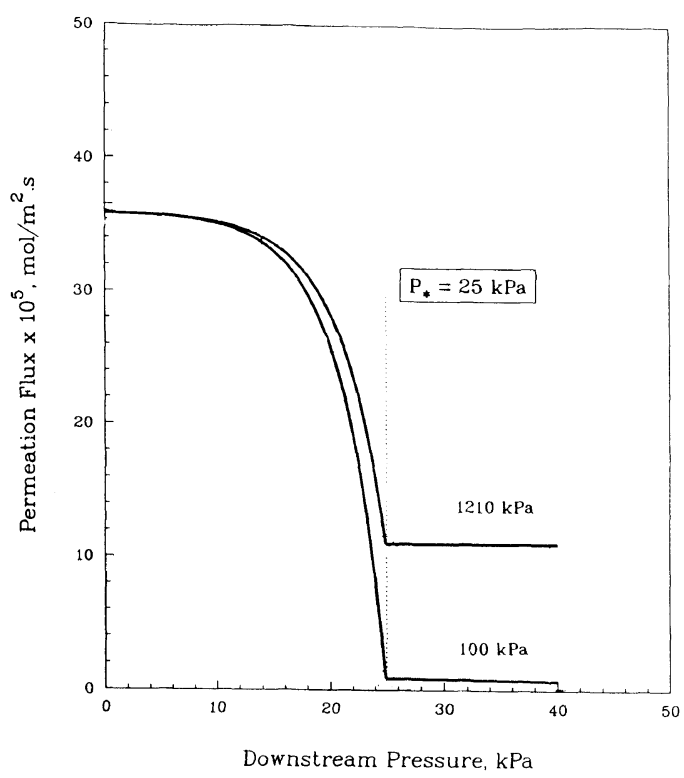

Figure 2. Effects of upstream and downstream pressure on the pervaporation flux by the transport equations of Greenlaw et al. ${ }^{5}$

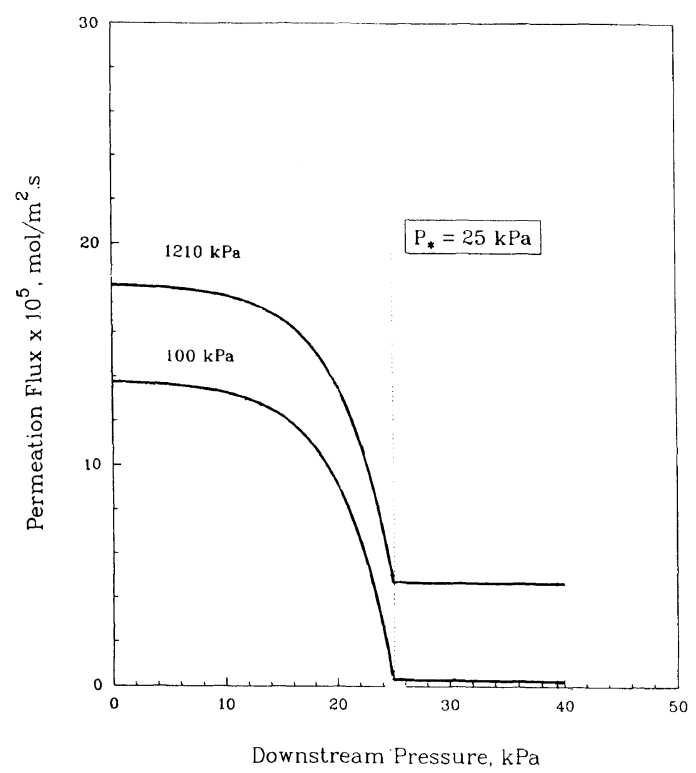

Figure 3. Effects of upstream and downstream pressure on the pervaporation flux by the newly developed transport model. 


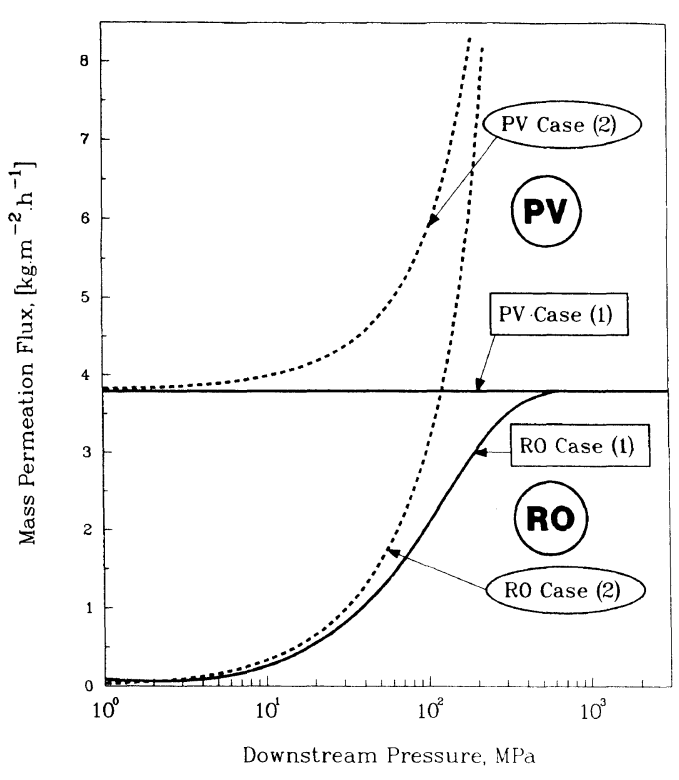

Figure 4. Effects of upstream and downstream pressure on the pervaporation flux by Kitaoka et al. ${ }^{12}$

$101,325 \mathrm{~Pa}$ ) for the following two cases.

1) The pressure of the permeant inside the membrane is constant and equal to the upstream pressure across the membrane.

2) The pressure of the permeant inside the membrane changes across the membrane from the upstream pressure to the downstream pressure linearly.

Although their assumption of permeant concentration and diffusion coefficient being constant across the membrane seems unrealistic, their conclusions, summarized below, seems to be valid.

1) In case 1), the upstream pressure has no effect on the pervaporation flux. The reverse osmosis flux approaches the pervaporation flux as the upstream pressure approaches infinity.

2) In case 2), the prevaporation flux increases and approaches infinity as the upstream pressure approaches infinity. The reverse osmosis flux also increases and becomes nearly equal to the pervaporation flux as the upstream pressure approaches infinity. One of their results is reproduced in Figure 4.

\section{RESULTS AND DISCUSSION}

Electron micrographic observation of APA membranes revealed neither finger-like nor sponge-like structures that are usually prominent in the asymmetric membranes.

In order to examine whether the membranes used in the present work were free from pinholes, pervaporation separation of an aqueous acetic acid solution and reverse osmosis separation of an aqueous sodium chloride solution were conducted for the PDMS membrane and the APA membrane, respectively. Pervaporation and reverse osmosis experiments were considered best to examine the absence of pinholes, particularly when one side of the membrane was in contact with the feed liquid and in a considerably swollen state. Using the PDMS membrane, acetic acid mole fraction increased from 0.079 in the feed to 0.126 in the product, which is in agreement with our previous result. ${ }^{14}$ Reverse osmosis separation of sodium chloride solute by the APA membrane, on the other hand, was $44 \%$, which value seems considerably lower than the separation obtained for an asymmetric reverse osmosis membrane of the same material. There is a considerable amount of information, however, that the sodium chloride separation of the dense homogeneous membrane is lower than that of the asymmetric membrane with respect to cellulose acetate material, ${ }^{11}$ probably due to difference in the alignment of the polymer segment in the dense active layer of the asymmetric membrane from that of the thick homogeneous membrane. Therefore, the above sodium chloride separation was considered acceptable for the dense homogeneous membrane of APA material. Figure 5 illustrates the results of pervaporation experiments for the system water-PDMS membrane. Three series of experiments were conducted; i.e., the upstream pressure was maintained at 101.325 $\mathrm{kPa}$ (0 psig, or atmospheric pressure), $446 \mathrm{kPa}$ (50 psig), and $791 \mathrm{kPa}$ (100 psig), respectively, while the downstream pressure was changed 


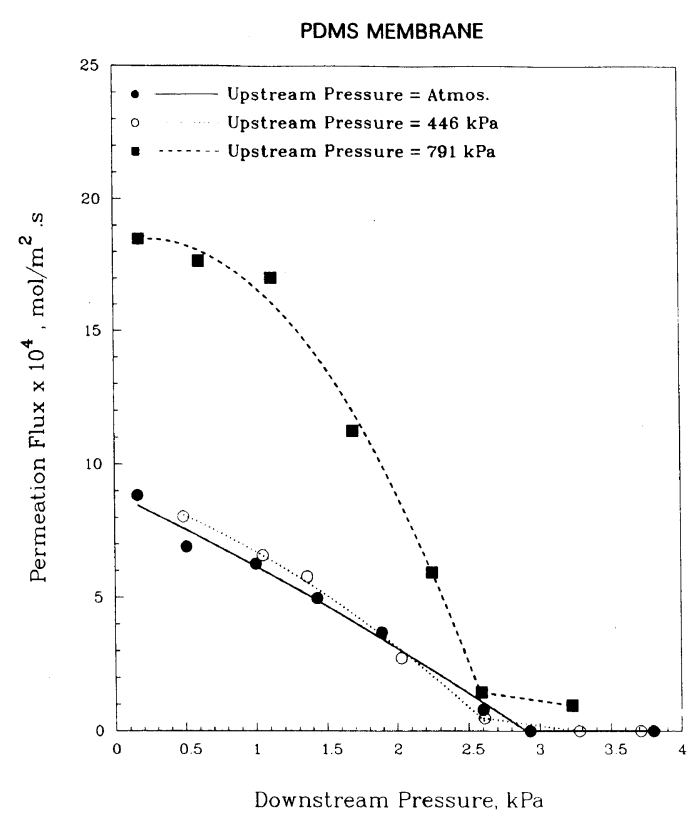

Figure 5. Experimental pervaporation data with respect to PDMS membranes.

in the range $0.133-4 \mathrm{kPa}(1-30 \mathrm{mmHg})$. It should be noted that a high initial permeation rate was observed when a higher than atmospheric pressure was applied on the upstream side of the membrane, while the downstream side was maintained at an atmospheric pressure. The permeation rate, however, started to decrease when vacuum was applied on the downstream side until a steady state was reached. Figure 5 presents the permeation rate data obtained after the steady state was achieved. These experimental results can be summarized as follows.

1) When the upstream pressure was an atmospheric pressure, practically no permeation flux was observed at downstream pressures above the saturation vapor pressure of water $\left(2.8 \mathrm{kPa}\right.$ or $21 \mathrm{mmHg}$ at $\left.23^{\circ} \mathrm{C}\right)$. Pervaporation flux increased parabolically with a decrease of the downstream pressure when the latter pressure was blow the saturation vapor pressure of water, confirming the tendency that has been observed previously by different authors. ${ }^{5,6}$ Inflection points are given in Figure
5 at 2.6 to $2.9 \mathrm{kPa}$, depending on the upstream pressure. It is difficult to say if three was a shift of the inflection point from the saturation vapor pressure. Such a shift was attributed to permeant-membrane interaction. ${ }^{14,15}$

2) When the upstream pressure was 446 $\mathrm{kPa}$, the permeation flux was nearly equal to that of an atmospheric pressure in the entire range of the downstream pressure.

3) When the upstream pressure was 791 $\mathrm{kPa}$, very low but above zero permeation fluxes were observed at downstream pressures higher than the saturation vapor pressure. When the downstream pressure was lower than the saturation vapor pressure, the pervaporation fluxes were much greater than the ones observed at lower upstream pressures.

Figure 6 presents the data on permeation of water through an APA membrane. Four series of experiments were conducted; i.e., the upstream pressure was maintained at 101, 446, 791 , and $1136 \mathrm{kPa}$, respectively, while the downstream pressure was changed in the range $0.4-5.1 \mathrm{kPa}(3-38 \mathrm{mmHg})$. The main features of the experiments can be summarized as follows.

1) When the upstream pressure was maintained at an atmospheric pressure, the permeation flux was low at downstream pressures higher than the saturation vapor pressure of water $(2.8 \mathrm{kPa}$ or $21 \mathrm{mmHg}$ at $23^{\circ} \mathrm{C}$ ). With a further decrease of the downstream pressure pervaporation flux increased parabolically. Inflection point appeared at $2.4 \mathrm{kPa}$, which is less than the saturation vapor pressure.

2) When the upstream pressure was increased from $101 \mathrm{kPa}$ (atmospheric pressure) to $446 \mathrm{kPa}$, a significant increase in the permeation flux from that of the lower upstream pressure was observed at downstream pressures higher than the saturation vapor pressure. With a further decrease of the downstream pressure the pervaporation flux increased parabolically but only moderately. As a result the pervaporation flux at the lowest 


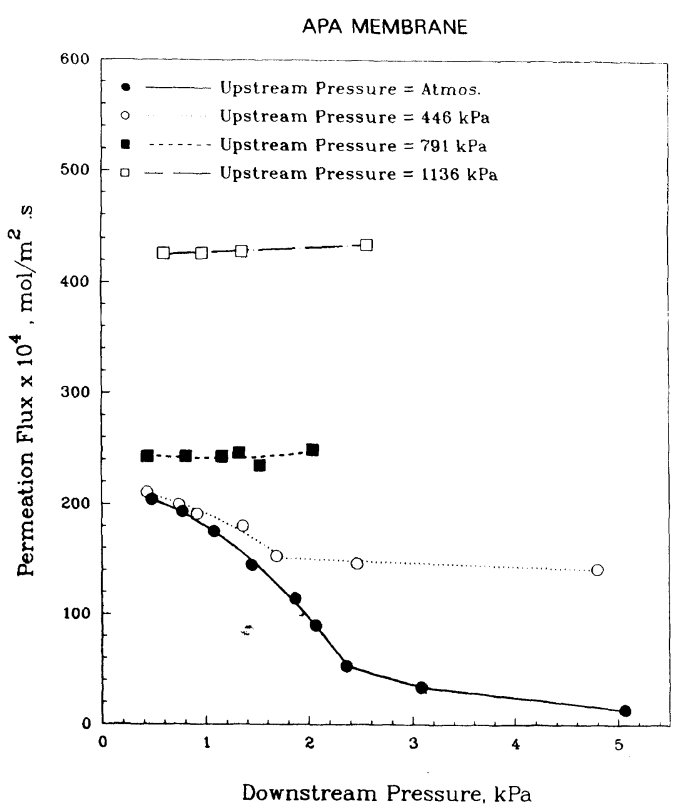

Figure 6. Experimental pervaporation data with respect to APA membranes.

downstream pressure was almost equal for upstream pressures of $101 \mathrm{kPa}$ and $446 \mathrm{kPa}$. The inflection point appeared at $1.7 \mathrm{kPa}$. Contrary to the previous work, ${ }^{14,15}$ the inflection points are at the pressures lower than the saturation vapor pressure. Neither permeant-membrane interaction nor the transport model can explain this phenomena.

3) When the upstream pressure was 791 $\mathrm{kPa}$, a further increase in permeation flux (from the permeation flux corresponding to the upstream pressure of $446 \mathrm{kPa}$ ) was observed at the downstream pressures higher than the saturation vapor pressure. The pervaporation flux was almost constant in the entire range of downstream pressure.

4) The tendency observed at the upstream pressure of $1136 \mathrm{kPa}$ was the same as that for $791 \mathrm{kPa}$.

Some of the data in Figures 5 and 6 were replotted in Figure 7 as the permeation flux versus the upstream pressure. In Figure 7 the pervaporation line represents the data obtained at downstream pressures ranging from 333 to

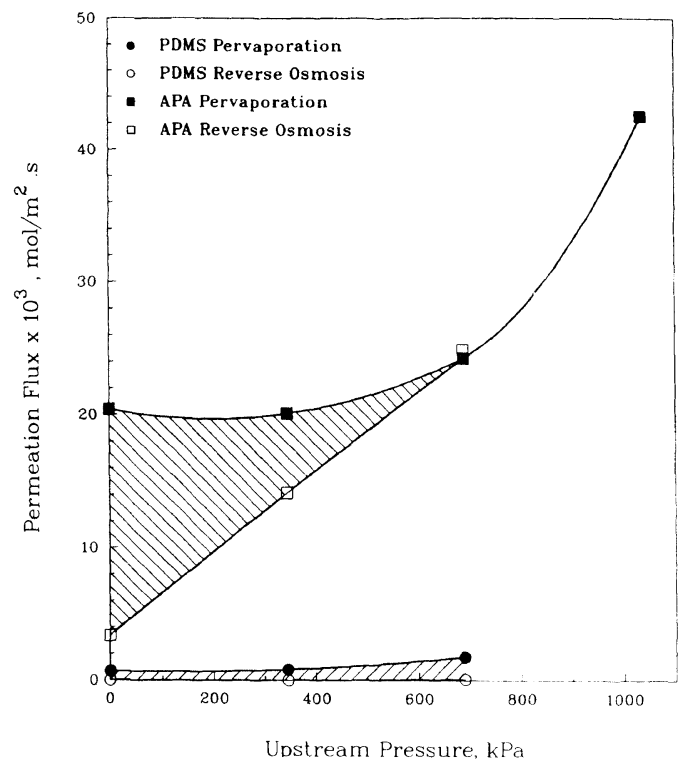

Figure 7. Flux versus upstream pressure for PDMS and APA membranes.

$613 \mathrm{~Pa}$ ( 2.5 to $4.6 \mathrm{mmHg}$ ), while the reverse osmosis line represents data obtained at downstream pressures above $2.8 \mathrm{kPa}$.

Comparing the shape of the curves experimentally obtained (Figures 5 and 6 ) with those predicted by transport theories (Figures 2 and 3 ), it is obvious that neither of the transport theories represents experimental data well. In particular, the assumption of the constant permeant pressure across the membrane predicts that a change in the upstream pressure has no effect on the pervaporation flux at zero downstream pressure, which contradicts the experimental results for both PDMS and APA membranes. Apparently, the experimental data for APA shown in Figure 7 have closest resemblance to the case (2) of Kataoka et al. However, the upstream pressure at which pervaporation flux meets with the reverse osmosis flux in Figure 7 is much lower than the prediction by Kataoka et al. ${ }^{13}$

\section{CONCLUSIONS}

The following conclusions can be drawn 
from the present investigations.

1) The transport equations newly developed for pervaporation allow the permeant pressure change across the membrane and include liquid as well as vapor transport. These equations predict that the pervaporation flux (at the downstream pressure close to zero $\mathrm{Pa}$ ) increases linearly with an increase in the upstream pressure. The effect of upstream pressure on pervaporation rate was also predicted by the pore model.

2) The solution-diffusion model predicts no upstream pressure effect on the permeation flux (at the downstream pressure close to zero $\mathrm{Pa}$ ) when the permeant pressure across the membrane is assumed to be constant and equal to the upstream pressure. The upstream pressure may have an effect on the pervaporation flux when there is a pressure change across the membrane.

3) Experimental data on water permeation through PDMS and APA membranes showed an increase in the pervaporation flux with an increase in the upstream pressure. However, the tendency observed in the experimental data agrees neither of transport models.

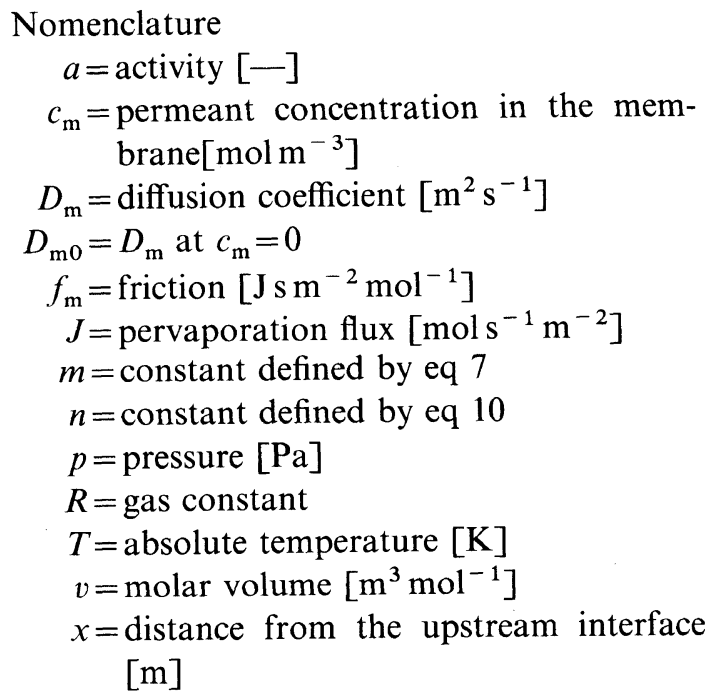

Greek letters

$\delta=$ effective membrane thickness [m] $\delta_{\mathrm{a}}=$ thickness of the layer where liquid transport prevails [m]

$\delta_{\mathrm{b}}=$ thickness of the layer where vapor transport prevails $[\mathrm{m}]$

$\zeta=p / p_{*}$

$\mu=$ chemical potential $\left[\mathrm{J} \mathrm{mol}^{-1}\right]$

$\sigma=$ constant defined by eq 7

$\tau=$ constant defined by eq 7

Subscript

$2=$ properties of feed

$3=$ properties of permeate

* = properties of saturated vapor

\section{REFERENCES}

1. J. Hauser, A. Heintz, G. A. Reinhardt, B. Schmittecker, M. Wesslein, and R. N. Richtenthaler, "Sorption, Diffusion, and Pervaporation of Water/ Alcohol Mixtures in Poly(vinyl alcohol) (PVA)Membranes. Experimental Results and Theoretical Treatment," in "Proceedings of Second International Conference on Pervaporation Processes in the Chemical Industry," R. Bakish, Ed., Bakish Materials Corp., Englewood, NJ, 1987, pp 15-33.

2. Y. M. Lee, D. Bourgeois, and G. Belfort, "Selection of Polymer Membrane Materials for Pervaporation," in "Proceedings of Second International Conference on Pervaporation Processes in the Chemical Industry," R. Bakish, Ed., Bakish Materials Corp., Englewood, NJ, 1987, pp 249-265.

3. J. Neel, "Fundamentals and General Chemical Engineering Aspects of Pervaporation," in "Proceedings of Firtst International Conference on Pervaporation Processes in the Chemical Industry," R. Bakish, Ed., Bakish Materials Corp., Englewood, NJ, 1986, pp 10-40.

4. J. W. F. Spitzen, E. Elsinghorst, M. H. V. Mulder, and C. A. Smolders, "Solution-Diffusion Aspects in the Separation of Ethanol/Water Mixtures with PVA Membranes," in "Proceedings of Second International Conference on Pervaporation Processes in the Chemical Industry," R. Bakish, Ed., Bakish Materials Corp., Englewood, NJ, 1987, pp 209-224.

5. F. W. Greenlaw, W. D. Prince, R. A. Shelden, and T. V. Thompson, "Dependence of Diffusive Permeation Rates on Upstream and Downstream Pressures. I. Single Component Permeant," J. Membrane Sci., 2, 141 (1977).

6. T. Okada and T. Matsuura, "A New Transport Model for Pervaporation," J. Membrane Sci., 59, 133 (1991).

7. T. Okada, M. Yoshikawa, and T. Matsuura, "A 
Study on the Pervaporation of Ethanol/Water Mixtures on the Basis of Pore Flow Model, $J$. Membrane Sci., 59, 151 (1991).

8. T. Okada and T. Matsuura, "Predictability of Transport Equations for Pervaporation on the Basis of Pore Flow Mechanism," J. Membrane Sci., 70, 163 (1992).

9. D. R. Paul and O. M. Ebra-lima, "The Mechanism of Liquid Transport through Swollen Polymer Membranes," J. Appl. Polym. Sci., 15, 2199 (1971).

10. U. Merten, "Transport Properties of Osmotic Membranes," in "Desalination by Reverse Osmosis," U. Meten, Ed., The M.I.T. Press, Cambridge, Massachusetts, 1966, pp 15-54.

11. H. K. Lonsdale, "Properties of Cellulose Acetate Membranes," in "Desalination by Reverse Osmosis," U. Merten, Ed., The M. I. T. Press, Cambridge, Massachusetts, 1966, pp 93-160.
12. C. H. Lee, "Theory of Reverse Osmosis and Other Membrane Permeation Operations," J. Appl. Polym. Sci., 19, 83 (1975).

13. T. Kataoka, T. Tsuru, S. Nakao, and S. Kimura, "Membrane Transport Properties of Pervaporation and Vapor Permeation in Ethanol-Water System Using Polyacrylonitrile and Cellulose Acetate Membranes," J. Chem. Eng. Jpn., 24, 334 (1991).

14. M. Yoshikawa, T. Matsuura, and D. Cooney, "Studies on the State of Permeant in the Membrane and Its Effect on Pervaporation Phenomena," J. Appl. Polym. Sci., 42, 1417 (1991).

15. M. Yoshikawa and T. Matsuura, "Studies on the State of Methanol in a Polydimethylsiloxane Membrane by Differential Scanning Calorimetry and Pervaporation Technique," Polym. J., 23, 1025 (1991). 